Research Article

\title{
Dynamic Carrying Capacity Analysis of Double-Row Four-Point Contact Ball Slewing Bearing
}

\author{
Yunfeng Li and Di Jiang \\ School of Mechatronics Engineering, Henan University of Science and Technology, Luoyang 471003, China \\ Correspondence should be addressed to Yunfeng Li; liyunfeng379@126.com
}

Received 5 May 2015; Revised 31 August 2015; Accepted 16 September 2015

Academic Editor: Michael Vynnycky

Copyright ( $) 2015$ Y. Li and D. Jiang. This is an open access article distributed under the Creative Commons Attribution License, which permits unrestricted use, distribution, and reproduction in any medium, provided the original work is properly cited.

\begin{abstract}
Carrying capacity is the most important performance index for slewing bearings. Maximizing the carrying capacity of slewing bearing is one pursuing goal for the bearing designer; this is usually realized by optimizing the design parameters. A method of analyzing the carrying capacity of double-row four-point contact ball slewing bearing by using dynamic carrying capacity surfaces was proposed in this paper. Based on the dynamic load carrying capacity surface of the slewing bearing, the effect of changes of the bearing design parameters, such as axial clearance, raceway groove radius coefficient, and contact angle, on the dynamic carrying capacity of the slewing bearing was researched; the trend and the degree of the effect of the micro changes of the bearing design parameters on the dynamic load carrying capacity of the bearing were discussed, and the results provide the basis for optimizing the design parameter of this type of slewing bearing.
\end{abstract}

\section{Introduction}

Slewing bearings have special structure and can carry combined radial, axial, and tilting moment loads simultaneously; they are widely used in the slewing arrangement of lift, metallurgy, mining, construction, harbor and wind turbine machinery, and so forth. Maximizing the carrying capacity of the slewing bearing is one pursuing goal for the bearing designer, and understanding the influence of design parameters on the carrying capacity of the bearing is needed, so that reasonable value of the design parameters can be selected.

In recent years, mechanics calculation problem of slewing bearing has attracted much attention from the researchers. Amasorrain et al. [1] established the statics equilibrium equations of the single-row four-point contact ball slewing bearing, and the rolling element loads were obtained by solving the equations. Potočnik et al. [2] established the equilibrium equations of the double-row four-point contact ball slewing bearing by using vectors to represent the bearing geometrical structure, and the maximum rolling element load was obtained by solving the equations. Olave et al. [3] proposed a procedure for obtaining the load distribution in a single-row four-point contact slewing bearing considering the effect of the structure's elasticity; the result shows that the contact force obtained on the most loaded rolling element is different for the supporting structures with different shapes. Daidié et al. [4] presented a 3D finite element simplified analysis of load distribution and contact angle variation in a slewing ball bearing; the rolling elements were modeled by nonlinear traction springs. The influence of the most important parameters such as the contact angle, the stiffness of the bearings, and the supporting structure is discussed. The above research works were conducted around the calculation of the rolling element load distribution of the slewing bearing mainly.

Gao et al. [5] established the nonlinear equations of single-row four-point contact slewing bearing and discussed the sensitivity of each parameter influencing the maximum ball contact force and the bearing service life. Chen et al. [6] developed a mechanical model for double-row four-point contact ball bearing and analyzed the effects of the geometric parameters on the bearing load capacity which was represented by the maximum ball contact force. Although Gao et al. [5] and Chen et al. [6] all focused on the effects of geometric parameters on the carrying capability of the slewing bearing, their research was aimed at a given working load set; the result is only effective for this working condition. Aguirrebeitia et al. [7] presented a procedure for the assessment of 


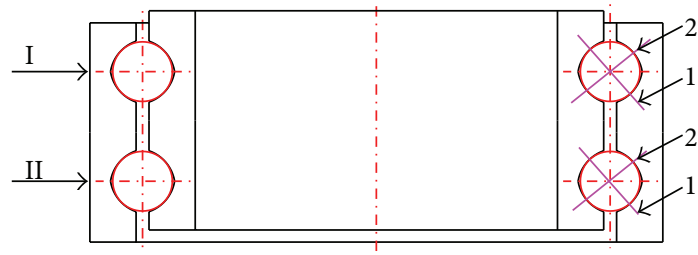

FIgURE 1: Raceways and contact pairs of bearing.

the limiting values of the loads acting on a four-point contact slewing bearing; the procedure results in a surface formed by a cloud of points in a three-dimensional coordinate system whose axes are the axial load, the radial load, and the tilting moment load. Aguirrebeitia's method focused on the static carrying capacity for the design of four-point contact slewing bearing.

The intention of this paper is to systematically research the effect of the changes of the geometrical parameters on the dynamic carrying capacity of the double-row four-point contact ball slewing bearing by using carrying capacity surfaces. The dynamic carrying capacity surfaces represent the ability of the slewing bearing to withstand the joint action of radial load, axial load, and tilting moment load in the whole 3D load domain, but not a given working load set. The effect of bearing geometrical parameters on the dynamic load carrying capacity of the bearing was researched by using dynamic carrying capacity surfaces, so as to use the law to guide the design of this kind of bearing.

\section{Geometrical Relationship in Bearing}

In the axial cross section of the bearing, the raceway of four-point contact ball slewing bearing is composed of two sections of arcs; the center of the arc is called groove curvature center. The loaded rolling elements contact with one section of the arc of inner ring raceway and one section of the arc of outer ring raceway in the opposite direction; these two contacts are called one contact pair. In this paper, the contact pair between the rolling element and the upper half of the inner ring raceway and the down half of the outer ring raceway is denoted by 1 ; the contact pair between the rolling element and the down half of the inner ring raceway and the upper half of the outer ring raceway is denoted by 2 . For the convenience of analysis, the upper row raceway is denoted by $\mathrm{I}$, and the bottom row raceway is denoted by II, as shown in Figure 1.

Before the bearing being loaded, according to the geometrical relationship of the bearing in Figure 2, the distance between the raceway groove curvature centers of the inner ring and the outer ring of one contact pair of any rolling element position is

$$
A=\left(f_{i}+f_{e}-1\right) D_{w}-G_{a} \sin \alpha_{0},
$$

where $f_{i}$ is the curvature radius coefficient of the inner raceway, $f_{e}$ is the curvature radius coefficient of the outer raceway, $D_{w}$ is the diameter of the rolling element, $G_{a}$ is the axial clearance of slewing bearing, and $\alpha_{0}$ is the nominal contact angle.

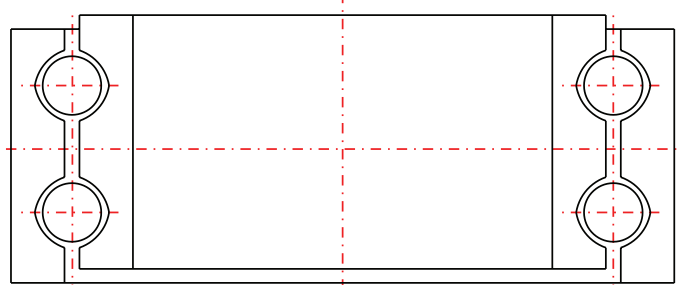

FIGURE 2: Bearing before being loaded.

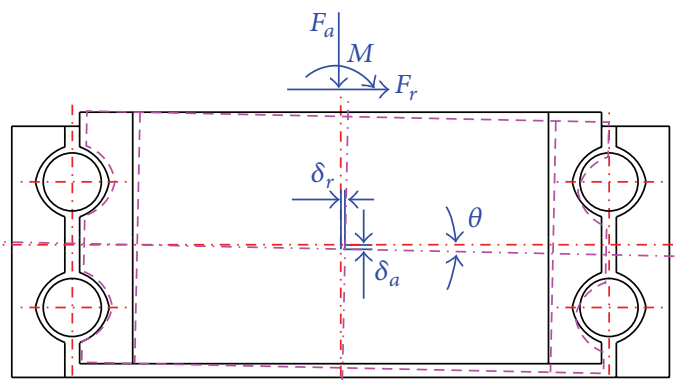

FIGURE 3: Inner ring displacement after the bearing being loaded.

In this case, the radius of locus of raceway groove curvature centers of inner ring can be expressed as

$$
\begin{aligned}
R_{i}= & 0.5 d_{m}+\left(f_{i}-0.5\right) D_{w} \cos \alpha_{0} \\
& -0.5 G_{a} \sin \alpha_{0} \cos \alpha_{0},
\end{aligned}
$$

where $R_{i}$ is the radius of locus of raceway groove curvature centers of inner ring and $d_{m}$ is the pitch diameter of slewing bearing.

As the bearing being loaded, suppose that the outer ring of the bearing is fixed. Under the combined action of radial load $F_{r}$, axial load $F_{a}$, and tilting moment load $M$, the corresponding radial displacement, axial displacement, and tilting angular displacement of the inner ring center $\operatorname{are} \delta_{r}, \delta_{a}$, and $\theta$, respectively, as shown in Figure 3 .

After the bearing being loaded, changes of all the distances between the groove curvature centers of the inner ring and the outer ring of contact pairs take place. For raceway I, the radial displacement of inner ring raceway revolution center relative to the outer raceway is

$$
\delta_{r \mathrm{I}}=\delta_{r}+0.5 d_{c} \theta,
$$

where $d_{c}$ is the axial distance between two rows of raceway grooves.

The distance between the groove curvature centers of the inner ring and the outer ring of contact pair 1 of any rolling element position is

$$
\begin{aligned}
S_{1 \mathrm{I} \psi}= & {\left[\left(A_{\mathrm{I}, 1} \sin \alpha_{0}+\delta_{a}+R_{i \mathrm{I}} \theta \cos \psi\right)^{2}\right.} \\
& \left.+\left(A_{\mathrm{I}, 1} \cos \alpha_{0}+\delta_{r \mathrm{I}} \cos \psi\right)^{2}\right]^{1 / 2} .
\end{aligned}
$$


The distance between the groove curvature centers of the inner ring and the outer ring of contact pair 2 of any rolling element position is

$$
\begin{aligned}
S_{2 \mathrm{I} \psi}= & {\left[\left(A_{\mathrm{I}, 2} \sin \alpha_{0}-\delta_{a}-R_{i \mathrm{I}} \theta \cos \psi\right)^{2}\right.} \\
& \left.+\left(A_{\mathrm{I}, 2} \cos \alpha_{0}+\delta_{r \mathrm{I}} \cos \psi\right)^{2}\right]^{1 / 2} .
\end{aligned}
$$

Similarly, for raceway II, the radial displacement of inner ring raceway revolution center relative to the outer raceway is

$$
\delta_{r \mathrm{II}}=\delta_{r}-0.5 d_{c} \theta
$$

The distance between the groove curvature centers of the inner ring and the outer ring of contact pair 1 of any rolling element position is

$$
\begin{aligned}
S_{1 \mathrm{II} \psi}= & {\left[\left(A_{\mathrm{II}, 1} \sin \alpha_{0}+\delta_{a}+R_{i \mathrm{II}} \theta \cos \psi\right)^{2}\right.} \\
& \left.+\left(A_{\mathrm{II}, 1} \cos \alpha_{0}+\delta_{r \mathrm{II}} \cos \psi\right)^{2}\right]^{1 / 2} .
\end{aligned}
$$

The distance between the groove curvature centers of the inner ring and the outer ring of contact pair 2 of any rolling element position is

$$
\begin{aligned}
S_{2 \mathrm{II} \psi}= & {\left[\left(A_{\mathrm{II}, 2} \sin \alpha_{0}-\delta_{a}-R_{i \mathrm{II}} \theta \cos \psi\right)^{2}\right.} \\
& \left.+\left(A_{\mathrm{II}, 2} \cos \alpha_{0}+\delta_{r \mathrm{II}} \cos \psi\right)^{2}\right]^{1 / 2} .
\end{aligned}
$$

At the same time, the external bearing load also caused the contact angle change of the bearing. For raceway I, use $\alpha_{1 \mathrm{I} \psi}$ to represent the operating contact angle of contact pair 1 at any rolling element position; then

$$
\begin{aligned}
\sin \alpha_{1 \mathrm{I} \psi} & =\frac{A_{\mathrm{I}, 1} \sin \alpha_{0}+\delta_{a}+R_{i \mathrm{I}} \theta \cos \psi}{S_{1 \mathrm{I} \psi}}, \\
\cos \alpha_{1 \mathrm{I} \psi} & =\frac{A_{\mathrm{I}, 1} \cos \alpha_{0}+\delta_{r \mathrm{I}} \cos \psi}{S_{1 \mathrm{I} \psi}} .
\end{aligned}
$$

Similarly, use $\alpha_{2 \mathrm{I} \psi}$ to represent the operating contact angle of contact pair 2 at any rolling element position; then

$$
\begin{aligned}
& \sin \alpha_{2 \mathrm{I} \psi}=\frac{A_{\mathrm{I}, 2} \sin \alpha_{0}-\delta_{a}-R_{i \mathrm{I}} \theta \cos \psi}{S_{2 \mathrm{I} \psi}}, \\
& \cos \alpha_{2 \mathrm{I} \psi}=\frac{A_{\mathrm{I}, 2} \cos \alpha_{0}+\delta_{r \mathrm{I}} \cos \psi}{S_{2 \mathrm{I} \psi}} .
\end{aligned}
$$

For raceway II, use $\alpha_{1 \mathrm{II} \psi}$ to represent the operating contact angle of contact pair 1 at any rolling element position; then

$$
\begin{aligned}
\sin \alpha_{1 \mathrm{II} \psi} & =\frac{A_{\mathrm{II}, 1} \sin \alpha_{0}+\delta_{a}+R_{i \mathrm{II}} \theta \cos \psi}{S_{1 \mathrm{II} \psi}}, \\
\cos \alpha_{1 \mathrm{II} \psi} & =\frac{A_{\mathrm{II}, 1} \cos \alpha_{0}+\delta_{r \mathrm{II}} \cos \psi}{S_{1 \mathrm{II} \psi}} .
\end{aligned}
$$

Use $\alpha_{2 \mathrm{II} \psi}$ to represent the operating contact angle of contact pair 2 at any rolling element position; then

$$
\begin{aligned}
\sin \alpha_{2 \mathrm{II} \psi} & =\frac{A_{\mathrm{II}, 2} \sin \alpha_{0}-\delta_{a}-R_{i \mathrm{II}} \theta \cos \psi}{S_{2 \mathrm{II} \psi}}, \\
\cos \alpha_{2 \mathrm{II} \psi} & =\frac{A_{\mathrm{II}, 2} \cos \alpha_{0}+\delta_{r \mathrm{II}} \cos \psi}{S_{2 \mathrm{II} \psi}}
\end{aligned}
$$

\section{Equilibrium Equations of Bearing}

The changes of groove curvature center distance cause the corresponding elastic contact deformations of contact pair 1 and contact pair 2 at any rolling element position. For raceway $\mathrm{I}$, the acting force between the rolling element and the raceway caused by the elastic contact deformation can be expressed as

$$
\begin{aligned}
& Q_{1 \mathrm{I} \psi}=K_{n}\left(S_{1 \mathrm{I} \psi}-A_{0}\right)^{3 / 2}, \\
& Q_{2 \mathrm{I} \psi}=K_{n}\left(S_{2 \mathrm{I} \psi}-A_{0}\right)^{3 / 2},
\end{aligned}
$$

where $K_{n}$ is the load-deflection factor.

For raceway II, the acting force between the rolling element and the raceway caused by the elastic contact deformation can be expressed as

$$
\begin{aligned}
& Q_{1 \mathrm{II} \psi}=K_{n}\left(S_{1 \mathrm{II} \psi}-A_{0}\right)^{3 / 2}, \\
& Q_{2 \mathrm{II} \psi}=K_{n}\left(S_{2 \mathrm{II} \psi}-A_{0}\right)^{3 / 2} .
\end{aligned}
$$

The inner ring is at an equilibrium state under the action of external loads $F_{r}, F_{a}$, and $M$ and all rolling element loads; the equilibrium equations can be obtained as follows:

$$
\begin{aligned}
F_{r} & =\left[\sum_{\psi=-\pi}^{\psi=+\pi}\left(Q_{1 \mathrm{I} \psi} \cos \alpha_{1 \mathrm{I} \psi}+Q_{1 \mathrm{II} \psi} \cos \alpha_{1 \mathrm{II} \psi}\right) \cos \psi\right. \\
& \left.+\sum_{\psi=-\pi}^{\psi=+\pi}\left(Q_{2 \mathrm{I} \psi} \cos \alpha_{2 \mathrm{I} \psi}+Q_{2 \mathrm{II} \psi} \cos \alpha_{2 \mathrm{II} \psi}\right) \cos \psi\right] \\
& =0 \\
F_{a} & -\left[\sum_{\psi=-\pi}^{\psi=+\pi}\left(Q_{1 \mathrm{I} \psi} \sin \alpha_{1 \mathrm{II} \psi}+Q_{1 \mathrm{II} \psi} \sin \alpha_{1 \mathrm{II} \psi}\right)\right. \\
& \left.-\sum_{\psi=-\pi}^{\psi=+\pi}\left(Q_{2 \mathrm{I} \psi} \sin \alpha_{2 \mathrm{I} \psi}+Q_{2 \mathrm{II} \psi} \sin \alpha_{2 \mathrm{II} \psi}\right)\right]=0,
\end{aligned}
$$




$$
\begin{aligned}
M & -\frac{1}{2} \\
& \cdot d_{m}\left[\sum_{\psi=-\pi}^{\psi=+\pi}\left(Q_{1 \mathrm{I} \psi} \sin \alpha_{1 \mathrm{I} \psi}+Q_{1 \mathrm{II} \psi} \sin \alpha_{1 \mathrm{II} \psi}\right) \cos \psi\right. \\
& \left.-\sum_{\psi=-\pi}^{\psi=+\pi}\left(Q_{2 \mathrm{I} \psi} \sin \alpha_{2 \mathrm{I} \psi}+Q_{2 \mathrm{II} \psi} \sin \alpha_{2 \mathrm{II} \psi}\right) \cos \psi\right] \\
& =0 .
\end{aligned}
$$

Substituting (1)-(14) into the above three equilibrium equations, a system of nonlinear equations can be obtained. If the geometrical parameters of the bearing are given, the values of the unknown variables $\delta_{r}, \delta_{a}$, and $\theta$ can be solved corresponding to a set of external loads $F_{r}, F_{a}$, and $M$, and the rolling element loads can be calculated further.

\section{Carrying Capacity Analysis}

4.1. Dynamic Load Carrying Capacity. The dynamic load carrying capacity of slewing bearing uses the maximum external load combination that the slewing bearing can withstand when the rating fatigue life of the slewing bearing is 30000 revolutions. The calculation of fatigue life of rolling bearing needs the rating rolling element load, the rolling element load distribution, and the equivalent rolling element load [8].

4.1.1. Rating Rolling Element Load. When the rating life of the ring is one million revolutions, the evenly distributed rolling element load that the bearing can carry is called rating rolling element load. Considering the influence of rolling element diameter on the rolling element loads, function $F\left(D_{w}\right)$ is introduced:

$$
\begin{aligned}
& \text { if } D_{w} \leq 25.4 \mathrm{~mm}, F\left(D_{w}\right)=D_{w}^{1.8} ; \\
& \text { if } D_{w}>25.4 \mathrm{~mm}, F\left(D_{w}\right)=3.647 D_{w}^{1.4} .
\end{aligned}
$$

Then, the rating rolling element load of the inner ring is

$$
\begin{aligned}
Q_{c i}= & 98.1\left(\frac{2 f_{i}}{2 f_{i}-1}\right)^{0.41} \frac{(1-\gamma)^{1.39}}{(1+\gamma)^{1 / 3}}\left(\frac{\gamma}{\cos \alpha_{0}}\right)^{0.3} \\
& \cdot F\left(D_{w}\right) Z^{-1 / 3} .
\end{aligned}
$$

The rating rolling element load of the inner ring is

$$
\begin{aligned}
Q_{c e}= & 98.1\left(\frac{2 f_{e}}{2 f_{e}-1}\right)^{0.41} \frac{(1+\gamma)^{1.39}}{(1-\gamma)^{1 / 3}}\left(\frac{\gamma}{\cos \alpha_{0}}\right)^{0.3} \\
& \cdot F\left(D_{w}\right) Z^{-1 / 3}
\end{aligned}
$$

where $\gamma=D_{w} \cos \alpha / d_{m}$.

4.1.2. Equivalent Rolling Element Load. When computing the rating rolling element loads of the ring, it is assumed that the rolling element loads are distributed evenly. But, in the normal case, the rolling element loads are not evenly distributed in the bearing. If under the condition of an assumed evenly distributed rolling element load, the life of the ring is the same as the life of the ring with the real load distribution, the evenly distributed rolling element load of this assumption is called equivalent rolling element load. The equivalent rolling element load of the ring which rotates relative to a load is

$$
Q_{e \mu}=\left(\frac{1}{Z} \sum_{j=1}^{Z} Q_{j}^{3}\right)^{1 / 3},
$$

where $Q_{j}$ is the ball-raceway normal load and $Z$ is the ball number of each row.

The equivalent rolling element load of the ring which keeps still relative to a load is

$$
Q_{e v}=\left(\frac{1}{Z} \sum_{j=1}^{Z} Q_{j}^{10 / 3}\right)^{3 / 10}
$$

The equivalents $Q_{e \mu 1}, Q_{e \nu 1}, Q_{e \mu 2}$, and $Q_{e \nu 2}$ of contact pairs 1 and 2 can be calculated by using the above two formulas.

4.1.3. Rating Fatigue Life Calculation. The rating fatigue life of the raceway which rotates relative to a load is

$$
L_{\mu}=\left(\frac{Q_{c \mu}}{Q_{e \mu}}\right)^{3} .
$$

The fatigue life of the raceway which keeps still relative to a load is

$$
L_{v}=\left(\frac{Q_{c v}}{Q_{e v}}\right)^{3}
$$

By using the above two formulas the fatigue life of the four raceways can be computed as $L_{\mu 1}, L_{\nu 1}, L_{\mu 2}$, and $L_{\nu 2}$, and the fatigue of the bearing is

$$
L=\left(L_{\mu 1}^{-1.11}+L_{\nu 1}^{-1.11}+L_{\mu 2}^{-1.11}+L_{\nu 2}^{-1.11}\right)^{-0.9}
$$

4.2. Dynamic Load Carrying Capacity. The calculation process of the dynamic load carrying capacity surface is as follows.

Step 1. Assign values to $F_{r}, F_{a}$, and $M$ continuously, for each set of values, using Newton-Raphson method to solve (15)(17) and calculate the values of $\delta_{r}, \delta_{a}$, and $\theta$ and calculate the rolling element load distribution further.

Step 2. Calculate the rating rolling element load and the equivalent rolling element load. The rating life of each raceway can be calculated by using rating rolling element load and the equivalent rolling element load, and the rating fatigue life of the whole bearing can be calculated further by (22)-(24).

Step 3. Compare the calculated life with 30000 revolutions; if the obtained life value is close to 30000 revolutions in a given scope, then these load sets $F_{r}, F_{a}$, and $M$ form a point on the dynamic load carrying capacity surface. 


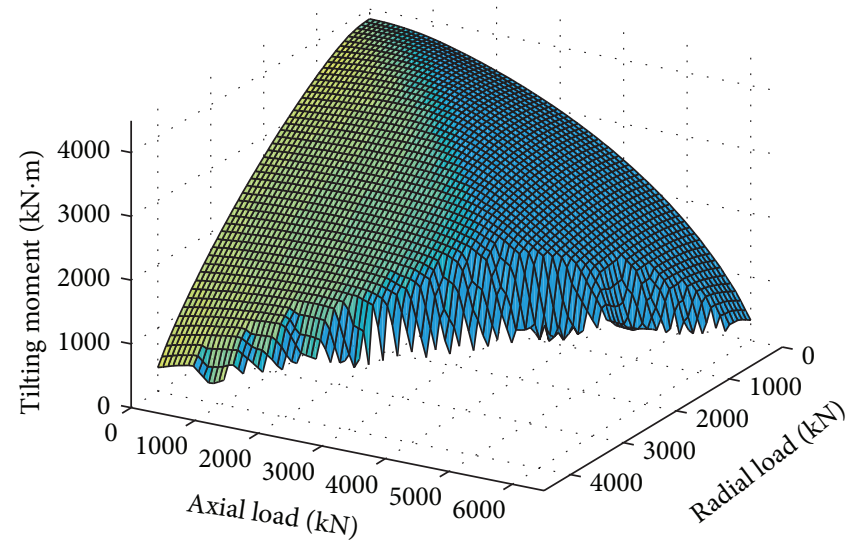

FIGURE 4: Dynamic carrying capacity surfaces of slewing bearing.
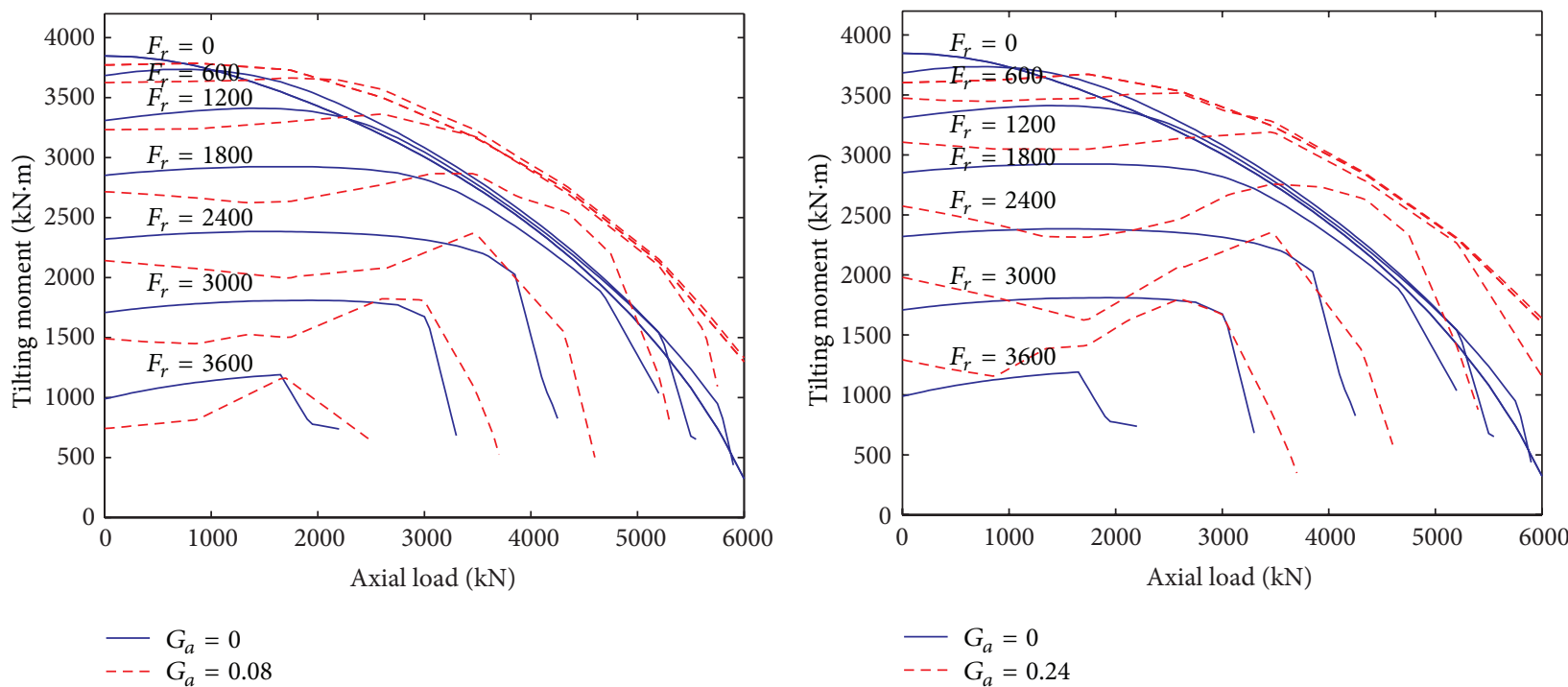

(a)

(b)

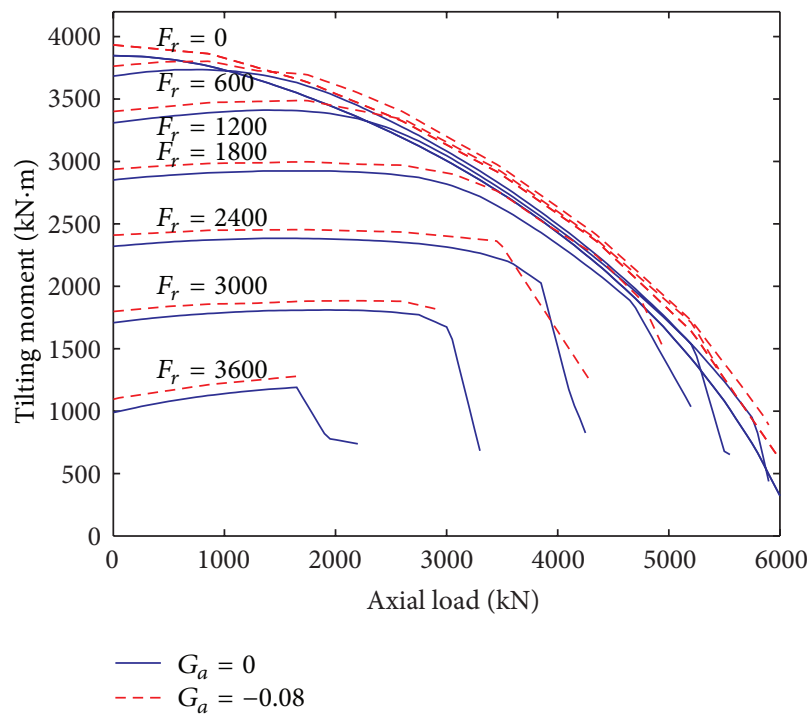

(c)

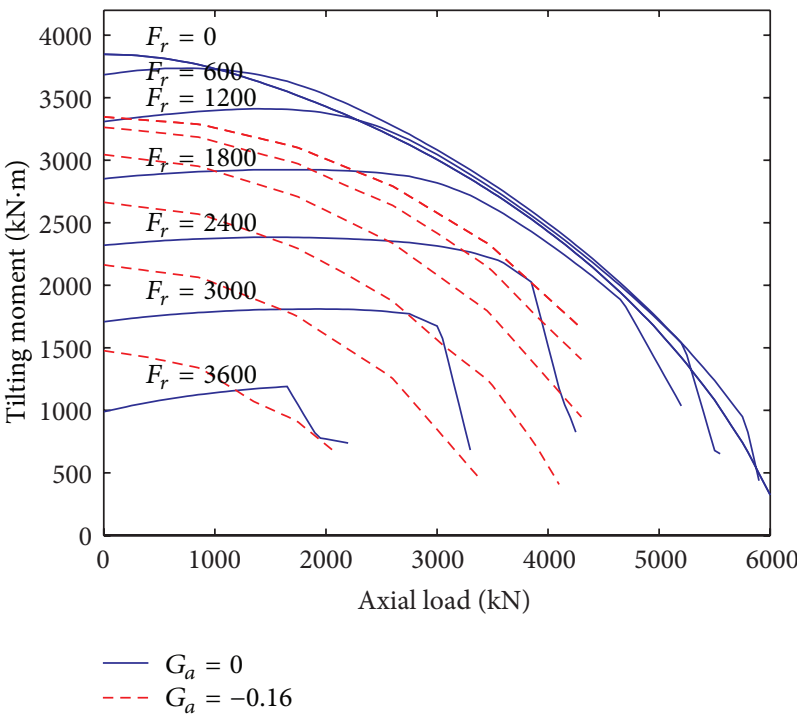

(d)

FIGURE 5: Effect of clearance on the carrying capacity. 


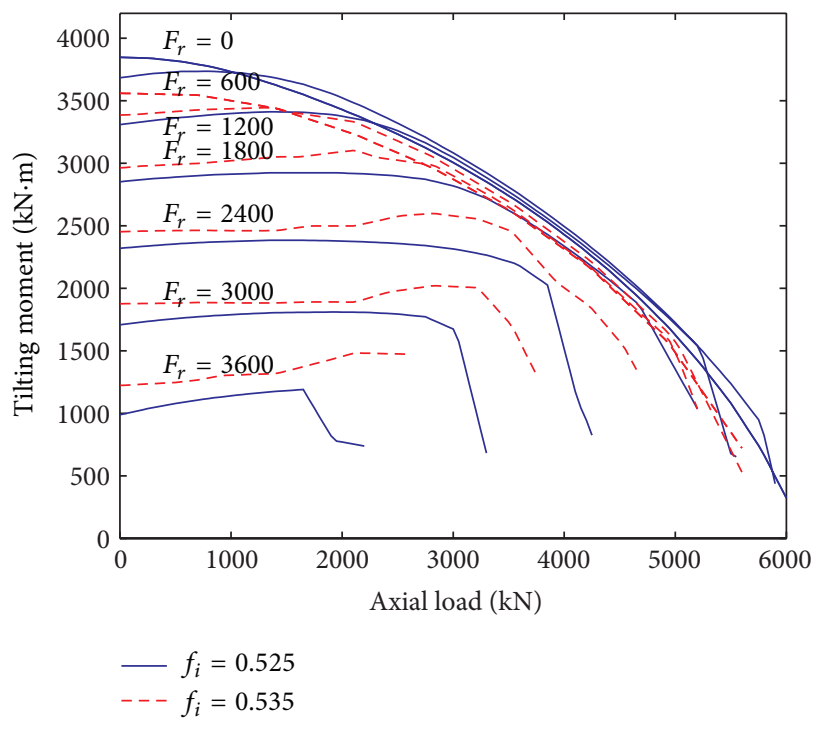

(a)

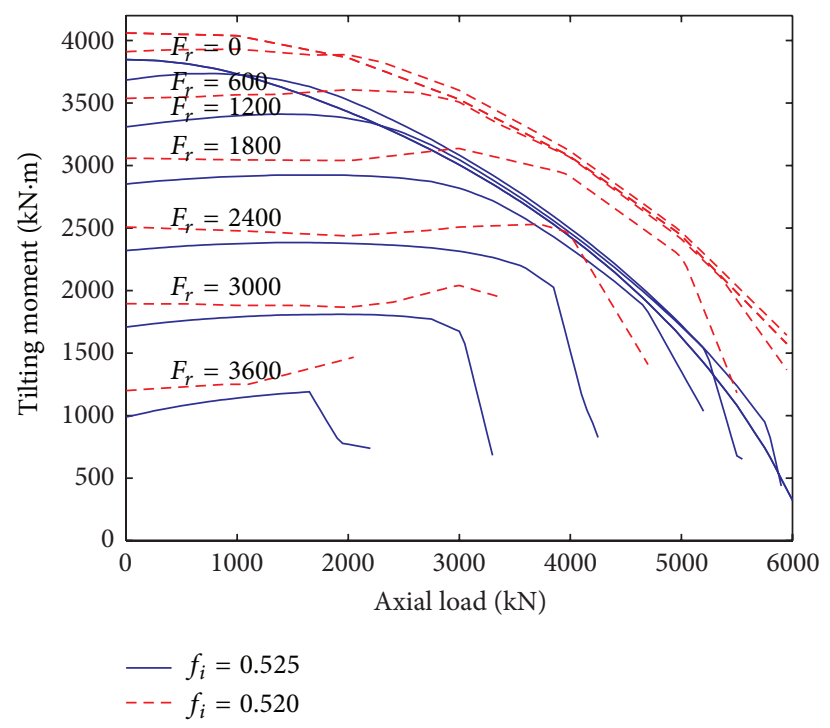

(b)

FiguRE 6: Effect of the groove curvature on the dynamic carrying capacity.

A certain type of double-row four-point contact ball slewing bearing used in the pitching system of $1.5 \mathrm{MW}$ wind turbine has the structure main parameters as follows: $d_{m}=$ 1900, $D_{w}=42.8625, d_{c}=58, \alpha=45^{\circ}, f_{i}=0.525, f_{e}=0.525$, and $Z=104$; the dynamic carrying capacity surfaces were obtained through calculation, as shown in Figure 4. As can be seen from the graph, with the increase of the radial load $F_{r}$, the relationship between the tilting moment $M$ and axial load $F_{a}$ changes with it.

\subsection{Effect of Geometrical Parameters on Carrying Capacity.} As bearing geometrical parameters change, the carrying capacity of the bearing changes with them, and the shape and the position of the corresponding dynamic load carrying capacity surface change with them. In order to research the effect of the geometrical parameters, such as clearance, raceway groove radius coefficient, and contact angle, on the dynamic carrying capacity of slewing bearing, take some cross sections of the carrying capacity surface corresponding to different radial loads to analyze; carrying curves corresponding to different radial loads were obtained; related results are shown in Figures 5-7.

As seen from the analyzed result, as the bearing parameter changes, for given radial load and axial load, the increased and decreased amplitudes of the allowed titling moment load reach to dozens of or several hundreds of "kNm." For different axial loads, the effect of the bearing parameter changes on the carrying capacity is different. When bearing external loads are given, applying these laws can fully improve the carrying capacity of the bearing.

4.3.1. Effect of Clearance. As can be seen from the results of Figure 5, when the clearance increases from 0 to 0.08 , the dynamic carrying capacity of the bearing decreases; when the clearance increases further to 0.24 , the dynamic carrying capacity of the bearing decreases obviously; when the clearance reduces from 0 to -0.08 , the dynamic carrying capacity of the bearing increases somewhat; when the clearance reduces further to -0.16 , the dynamic carrying capacity of the bearing decreases significantly. Therefore, moderate small negative clearance is most favorable for the dynamic carrying capacity of the bearing.

4.3.2. Effect of Groove Curvature. As can be seen from the results of Figure 6, when the groove curvature radius coefficient increases from 0.525 to 0.535 , the dynamic carrying capacity of the bearing decreases significantly; when the groove curvature radius coefficient decreases from 0.525 to 0.520 , the dynamic carrying capacity of the bearing increases significantly. Therefore, smaller groove radius curvature coefficients are most favorable for the carrying capacity of the bearing.

4.3.3. Effect of Contact Angle. As can be seen from the results of Figure 7, when the contact angle increases from $45^{\circ}$ to $50^{\circ}$, the dynamic carrying capacity of the bearing decreases, but the carrying capacity of the bearing increases somewhat as the axial load is larger. When the contact angle increases further to $60^{\circ}$, the dynamic carrying capacity of the bearing decreases significantly for smaller axial load, and the dynamic carrying capacity of the bearing increases significantly for larger axial load. Therefore, for the case of larger axial load, increasing contact angle is favorable for the dynamic carrying capacity of the bearing.

\section{Conclusion}

In this paper, through the research on the geometrical parameters of the double-row four-point contact ball slewing bearing, such as clearance, raceway groove radius coefficient, and contact angle, it is found that the micro changes of the bearing 


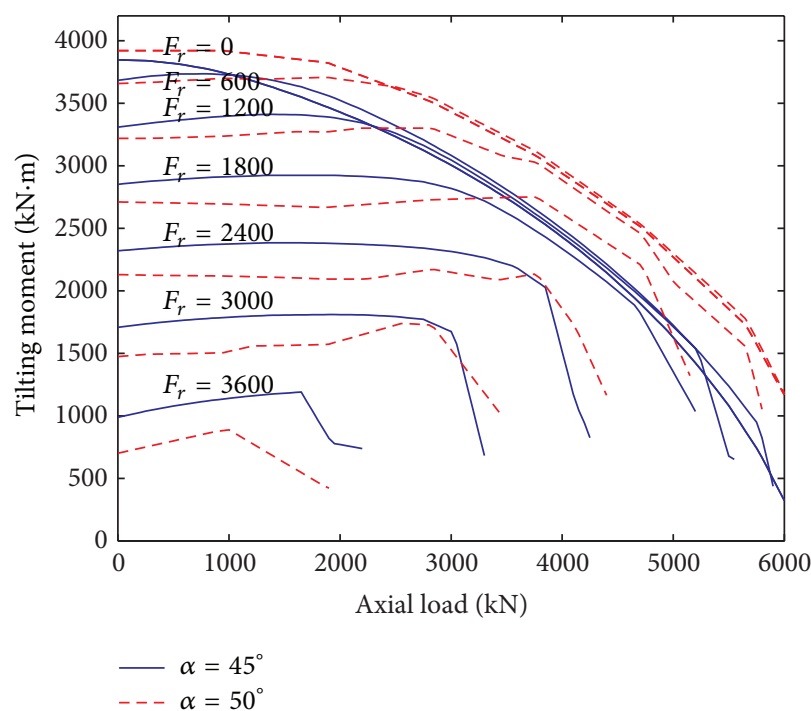

(a)

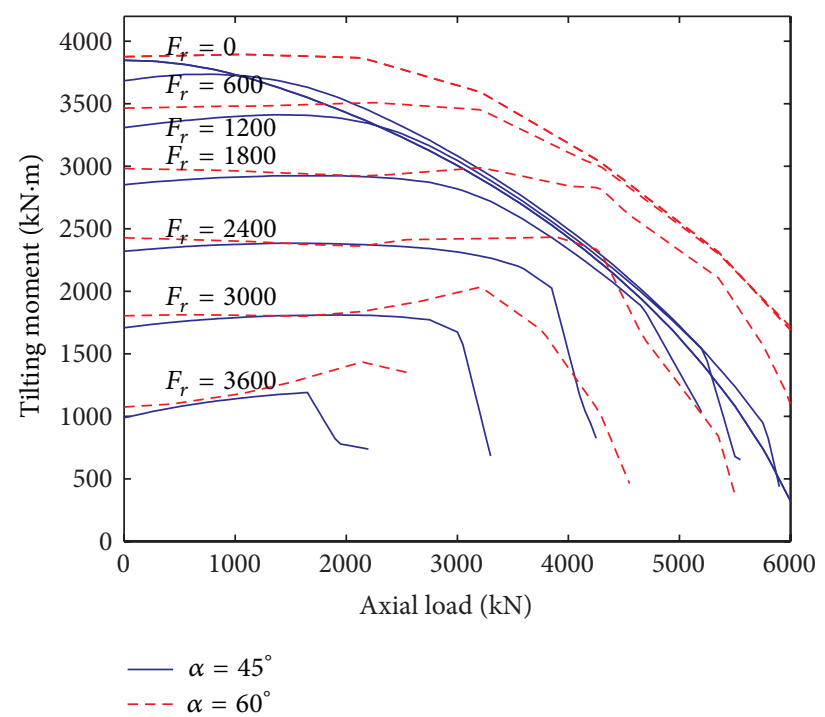

(b)

FIGURE 7: Effect of contact angle on the dynamic carrying capacity.

geometrical parameters have apparent effect on the dynamic carrying capacity of the bearing. The influence trends of the bearing design parameter changes on the dynamic load carrying capacity of the bearing are basically consistent. In general, appropriate small negative clearance is favorable for the dynamic carrying capacity of the bearing; the decrease of groove curvature radius coefficient is favorable for the dynamic carrying capacity of the bearing; when the relative value of the axial load of the bearing is larger, the increase of the contact angle is favorable for the dynamic carrying capacity of the bearing. These rules can be used to guide the design of this type of slewing bearing.

\section{Conflict of Interests}

The authors declare that there is no conflict of interests regarding the publication of this paper.

\section{References}

[1] J. I. Amasorrain, X. Sagartzazu, and J. Damián, "Load distribution in a four contact-point slewing bearing," Mechanism and Machine Theory, vol. 38, no. 6, pp. 479-496, 2003.

[2] P. Potočnik, P. Göncz, J. Flašker et al., "Fatigue life of double row slewing ball bearing with irregular geometry," Procedia Engineering, vol. 2, no. 1, pp. 1877-1886, 2010.

[3] M. Olave, X. Sagartzazu, J. Damian, and A. Serna, "Design of four contact-point slewing bearing with a new load distribution procedure to account for structural stiffness," Journal of Mechanical Design, vol. 132, no. 2, Article ID 021006, 2010.

[4] A. Daidié, Z. Chaib, and A. Ghosn, "3D simplified finite elements analysis of load and contact angle in a slewing ball bearing," Journal of Mechanical Design, vol. 130, no. 8, pp. 0823011-0826018, 2008.

[5] X. H. Gao, X. D. Huang, H. Wang, R. Hong, and J. Chen, "Effect of raceway geometry parameters on the carrying capability and the service life of a four-point-contact slewing bearing," Journal of Mechanical Science and Technology, vol. 24, no. 10, pp. $2083-$ 2089, 2010.

[6] G. C. Chen, P. Jia, and J. Q. He, "Effects of geometric parameters on static load capacity of a double-row four-point contact ball bearing," Journal of Mechanical Science and Technology, vol. 27, no. 4, pp. 1053-1061, 2013.

[7] J. Aguirrebeitia, R. Avilés, I. F. De Bustos, and M. Abasolo, "Calculation of general static load-carrying capacity for the design of four contact point slewing bearings," Journal of Mechanical Design, vol. 132, no. 6, pp. 064501-1-064501-6, 2010.

[8] T. A. Harris, Rolling Bearing Analysis, John Wiley \& Sons, New York, NY, USA, 4th edition, 2001. 


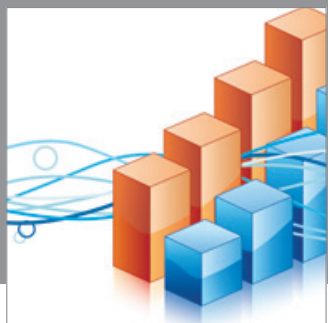

Advances in

Operations Research

mansans

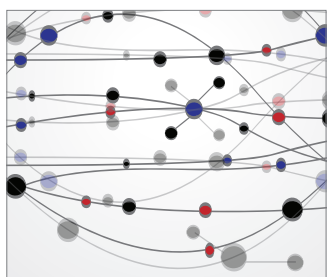

The Scientific World Journal
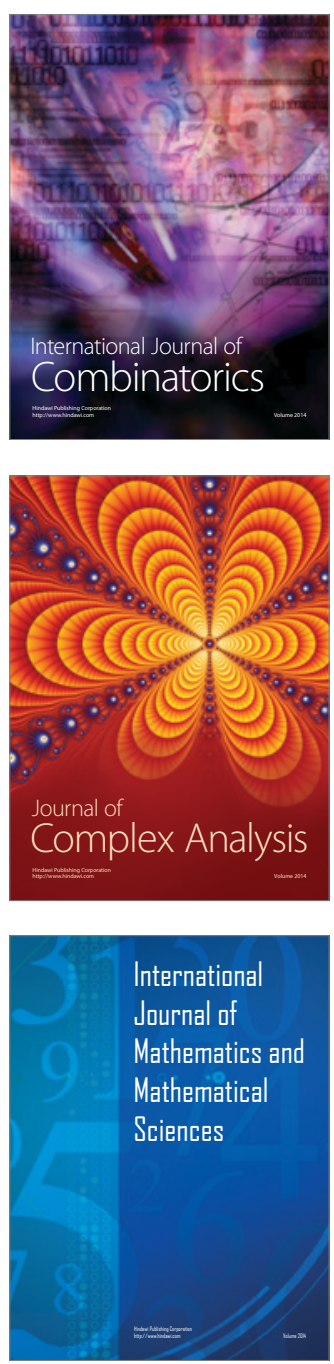
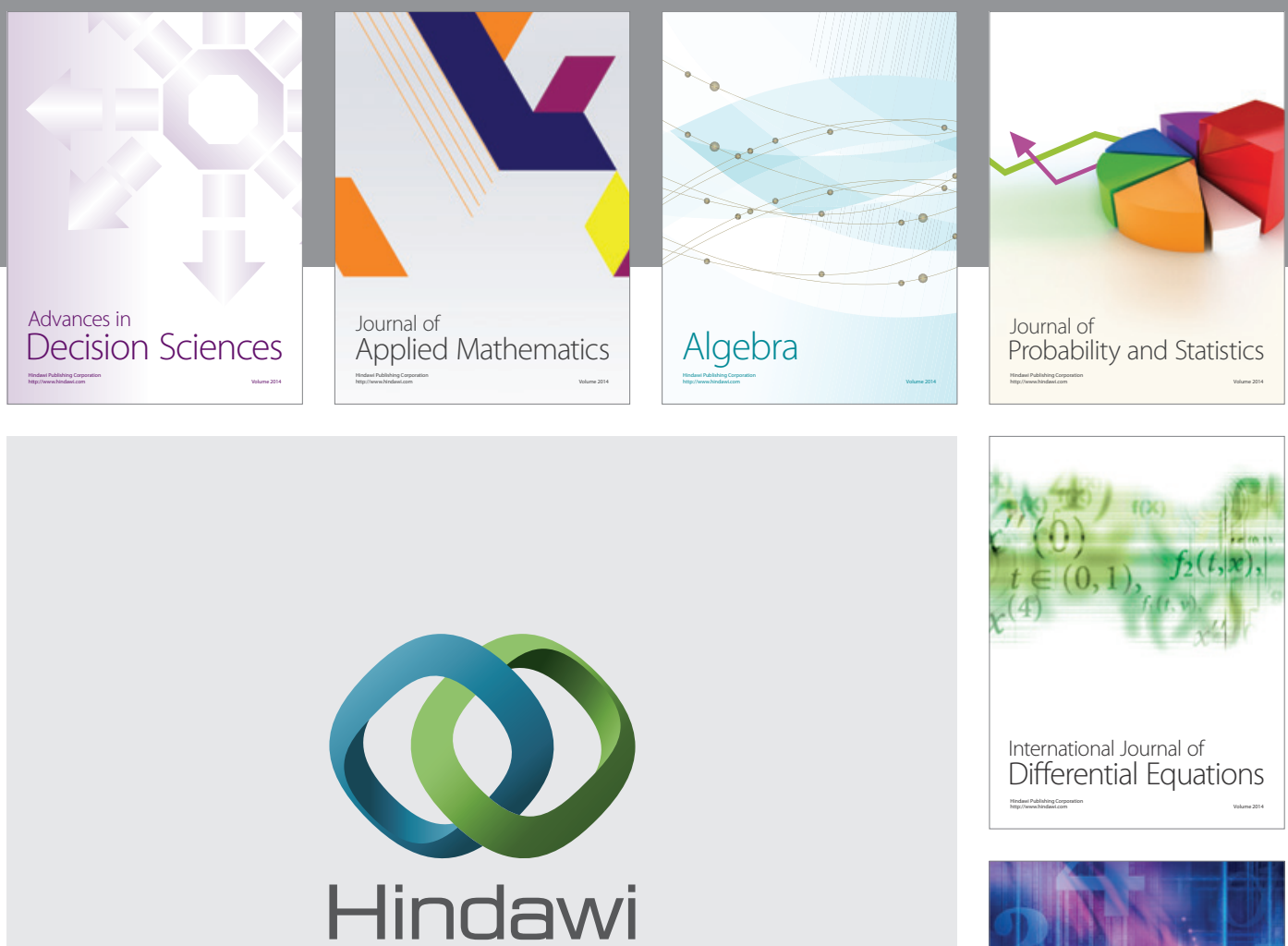

Submit your manuscripts at http://www.hindawi.com
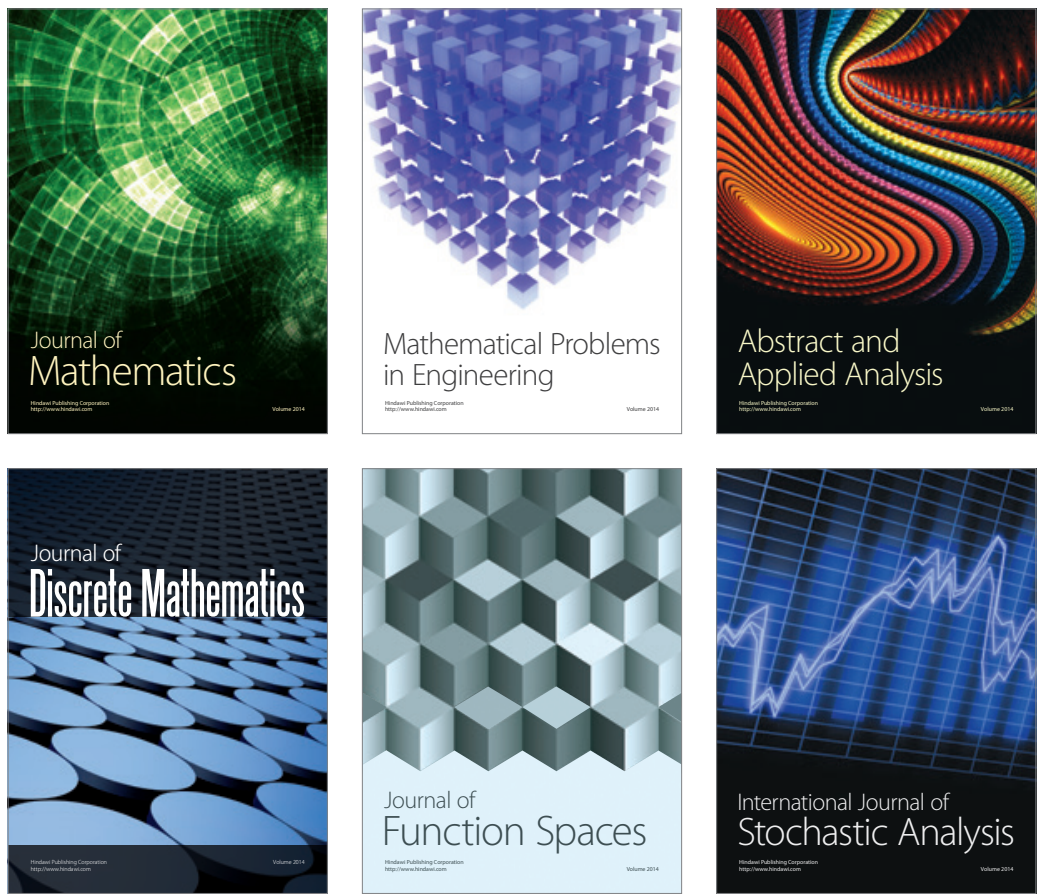

Journal of

Function Spaces

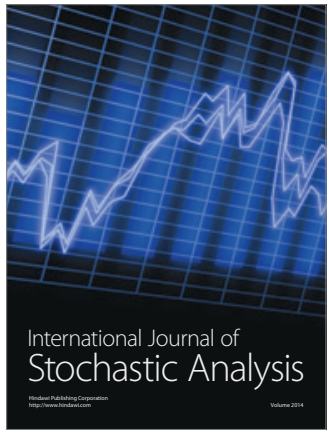

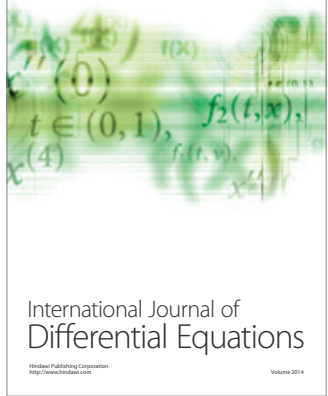
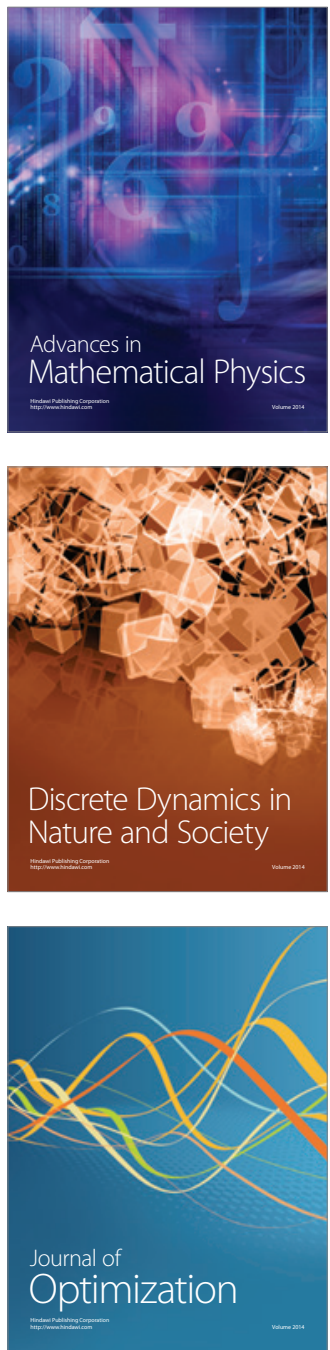